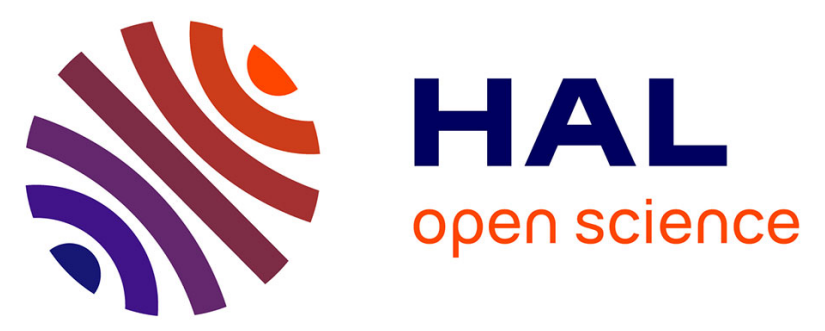

\title{
Les mutations du quartier de Saint-Romain-en-Gal et de l'îlot A
}

Jean-Luc Prisset, Laurence Brissaud

\section{To cite this version:}

Jean-Luc Prisset, Laurence Brissaud. Les mutations du quartier de Saint-Romain-en-Gal et de l'îlot A. Gallia - Fouilles et monuments archéologiques en France métropolitaine, 1994, Évolution urbaine à Saint-Romain-en-Gal: la rue du Commerce et la maison aux Cinq Mosaïques, 51, pp.118-129. 10.3406/galia.1994.3301 . hal-02468411

\section{HAL Id: hal-02468411 \\ https://hal.science/hal-02468411}

Submitted on 5 Feb 2020

HAL is a multi-disciplinary open access archive for the deposit and dissemination of scientific research documents, whether they are published or not. The documents may come from teaching and research institutions in France or abroad, or from public or private research centers.
L'archive ouverte pluridisciplinaire HAL, est destinée au dépôt et à la diffusion de documents scientifiques de niveau recherche, publiés ou non, émanant des établissements d'enseignement et de recherche français ou étrangers, des laboratoires publics ou privés.

\section{(이) $\$$}

Distributed under a Creative Commons Attribution - NonCommercial - NoDerivatives| 4.0 
[* s. avant J.-C. jusque sous Tibère. Dans le premier horizon, elles sont plus importantes que la sigillée avec essentiellement des imitations de formes précoces: dans le deuxième horizon, elles sont en concurrence avec la sigillée de la Gaule du Sud et des formes nouvelles apparaissent : imitations du service II. Il est arbitraire de se baser sur un aussi faible échantillonnage pour tracer une évolution de ces productions mais il semble qu'elles disparaissent dans la première moitié du I ${ }^{\mathbb{x}}$ s. après $\mathrm{J} . \mathrm{C}$.

La commune claire, cruches, pots et formes basses. avec une pâte calcaire fine sont des productions locales. Des formes similaires proviennent des ateliers de la «CNR» à Saint-Romain-en-Gal qui ne sont malheureusement pas datés par les données archéologiques. L'étude de notre matériel comme site de consommation permet de proposer une large période de production pour ces fours, de l'époque augustéenne au II $\mathrm{C} s$. La fabrication de pots avec un fin dégraissant, trouvés dans l'horizon 5 , n'est pas attestée sur le site.

Nous remarquons l'abondance des parois fines dans les contextes du $\mathrm{I}^{\mathrm{er}} \mathrm{s}$. qui sont en majorité des productions régionales, ainsi que la prédominance des gobelets dans l'horizon 1. Ce type de vaisselle est de moins en moins présent alors que celle en verre augmente, ce qui dénote dès la fin du $\mathrm{I}^{\mathrm{er}}$ et surtout au $\mathrm{II}^{\mathrm{e}} \mathrm{s}$. un changement dans les arts de la table.

\title{
LES MUTATIONS DU GUARTIER DE SAINT-ROMAIN-EN-GAL ET DE L'ÎLOT A
}

\author{
par Jean-Luc PRISSET et Laurence BRISSAUD
}

L'analyse de l'évolution de la Voie II et des transformations de la parcelle de la maison aux Cinq Mosaïques nous permettent de mieux cerner la genèse du quartier qu'avait révélée l'étude de la maison des Dieux Océans. Les informations essentielles livrées par le sondage de la rue, associées à une connaissance générale du site qui s'affine de plus en plus, notamment grâce à l'étude systématique de la voirie, contribuent largement à préciser le cadre du développement urbain. des origines jusqu'au début du $\mathrm{III}^{\mathrm{e}} \mathrm{s}$. après J.-C.

Par ailleurs, les transformations qui ont affecté les parcelles de l'îlot A permettent de se faire une idée plus précise des mutations foncières qui se sont opérées au cours des différentes époques. A partir d'exemples concrets, nous pourrons dégager quelques tendances concernant l'habitat.

\section{LE CADASTRE VIENNOIS}

Durant l'Age du Fer, nous savons, grâce aux études géomorphologiques (Bravard et alii, 1990): SavayGuerraz, Prisset, Delaval, 1993), que le site de SaintRomain-en-Gal est une île entourée par deux bras du Rhône. Cette situation s'achève à la fin de cette période du fait de l'enfoncement du fleuve dans son lit. Les eaux se concentrent à l'est de la vallée, dans un chenal profond, provoquant ainsi la condamnation du bras ouest. Dès lors, la mise hors d'eau de la plaine de SaintRomain-en-Gal favorise le développement d'une occupation humaine permanente.

\section{SAINT-ROMAIN-EN-GAL, SITE RURAL}

Au $I^{\mathrm{er}} \mathrm{s}$. avant J.-C., l'existence d'une borne plantée dans le terrain naturel limoneux vierge constitue l'un des premiers témoignages de cette présence humaine. Matérialisant une division de l'espace, elle nous indique qu'une délimitation de terrains a été effectuée en ces lieux et qu'une mise en culture a vraisemblablement débuté. Le fait que cette pierre présente une orientation est-ouest, qui va perdurer à travers toutes les transformations du quartier, nous incite à la considérer comme un élément appartenant à un découpage plus vaste qui dépasse le cadre du site.

La première approche concernant l'existence d'un cadastre viennois, réalisée en 1962, est restée très évasive, soulignant la faible empreinte laissée par celui-ci dans le paysage et suggérant comme cardo possible la route qui relie Feyzin à Vienne, sans plus d'explication (Chevallier, 1962). Une seconde étude, quoique très ponctuelle, a permis d'apporter des précisions plus nettes (Chouquer, Favory, 1980, p. 53-54). Ainsi, une centurie a été repérée sur la commune de SaintSymphorien-d'Ozon, entre les fermes du Grand et du Petit Chantoire, tandis que les axes moyens des chemins qui paraissent la délimiter ont fourni une orientation de $0,30^{\circ}$ ouest à ce cadastre ${ }^{1 / 4}$.

114 L'origine de la détermination de cette valeur de $0.30^{\circ}$ n'est pas explicitéc. 
Nous retrouvons une valeur proche sur le site de Saint-Romain-en-Gal avec la limite matérialiséc initialement par la borne. puis par les murs de clôture des parcelles (cf. supra, p. 13). Rapportée au nord, la déviation angulaire de cet alignement s'avère être de $5.30^{\circ}$ ouest. Cette valeur est certainement plus fiable que celle donnée précédemment dans la mesure où elle s'appuie sur une base antique concrète. Les cinq degrés de différence sont à mettre au compte des fluctuations liées à l'emplacement de la centurie repérée. Celle-ci est en effet située sur un bord de plateau, position qui a sans doute affecté les tracés des chemins. Par ailleurs. si nous prenons les différentes portions rectilignes de la route de Feyzin à Vienne comme références, nous observons une déclinaison variant de 1 à $4^{\circ}$ ouest, ce qui tend bien à prouver l'existence d'un lien entre tous ces éléments.

L'axe retrouvé à Saint-Romain-en-Gal apporte la confirmation du rattachement de ce cadastre à Vienne. tout en précisant son étendue. Nous savons dorénavant que le maillage couvre aussi la rive droite du Rhône, suivant en cela l'extension de la civitas. Nous pouvons tenter de définir la dimension de la centurie, malgré l'incertitude qui pèse sur les emplacements réels des limites. Si nous prenons comme référence la limite nord de la centurie du Petit Chantoire ${ }^{15}$. une taille de 704 ou $7(05 \mathrm{~m}$ apparaît. Il s'agit d'une valeur faible. quoique mesurée à partir de la plus grande distance possible, qui demanderait à être confirmée par une recherche plus systématique concernant l'étendue de cette cadastration.

La découverte du bornage de Saint-Romain-en-Gal. qui se trouve en relation stratigraphique avec l'évolution du quartier, offre la possibilité d'approcher la date de mise en place de cette centuriation. L'analyse du matériel issu des couches qui accompagnent le mur M9. à fondation de galets, place l'existence de celui-ci au début de l'époque augustéenne, soit dans les années 2010 avant J.-C.. date correspondant à celle de l'installation de l'état $1 \mathrm{~A}$ de la maison des Dieux Océans. Mais ces indices ne nous fournissent pas nécessairement l'époque de la construction de ce mur. Afin de préciser celle-ci. nous nous appuierons sur les types de maçonnerie rencontrés sur le site.

Lors de l'étude de la maison des Dieux Océans. l'hypothèse avait été faite que le mur nord. M333, lié au mortier blanc-gris (fig. 13, mur des sondages I 51 et I 66). pouvait être plus ancien que les quelques maçonneries dégagées au sud de la zone I. du côté de la Voie I (Desbat et alii. 1994 : chap. III - L’état IA. Le plan). Nous avons déjà mis en relief les différences de construction des murs qui jalonnent l'axe de cadastration. L'emploi de galets, matériau brut issu des

115 Il s'agirait de la dix-neuvième limites située au nord de l'axe de Saint-Romain-en-Gal. Elle passerait juste au sud de la ferme du Grand Chantoire. terrasses alluviales voisines, et la présence de fondations non cimentées confère une originalité à $\mathrm{M} 9$ qui le différencie nettement des constructions réalisées tout au long de l'évolution du site. Elle est l'expression d'un autre savoir-faire qui ne se retrouvera plus par la suite à Saint-Romain-cn-Gal. Malgré l'emploi d'un mortier de coloration blanc-jaune à jaune, nous avons donc tendance à lui conférer une antériorité sur l'ensemble des autres maçonneries, y compris celles réalisées avec un mortier blanc.

Les fouilles récentes de la place Triangulaire viennent confirmer cette hypothèse. Nous retrouvons en effet deux fondations perpendiculaires de galets imbriqués, non liés au mortier, marquant une limite parcellaire. Celles-ci ont été reprises comme fondations par des murs à mortier blanc-gris. Ainsi, M9 s'intègre dans un ensemble qui correspond aux plus anciennes constructions repérées sur le site.

Si nous nous référons au matéricl recueilli dans le comblement du fossé qui borde la Voie I au nord ${ }^{116}$. nous sommes en droit de supposer que la première occupation ayant laissé des indices matériels, murs ou céramiques, remonte aux années 40-30 avant J.-C. Dans la mesure où la borne précède le mur. nous pouvons envisager que la mise en place de la centuriation a eu lieu antérieurement à 40 . La faible valeur du module de vingt actus irait dans le sens d'une date haute (Chouquer, Favory, 1992, p. 103). Dès lors, sans trop de risque d'erreur, çette installation est à rapprocher de la déduction d'une colonie de droit latin à Vienne que les recherches de Chr. Goudineau permettent désormais de fixer aux années 46-45 (Goudineau. 1986, p. 176 : 1989, p. 23-36).

La plaine de Saint-Romain-en-Gal s'avère ainsi propice au développement de cultures au moins dès le milieu du Ier $s$. avant J.-C. Nous pouvons en effet penser que ce secteur n'aurait pas été cadastré si des conditions de salubrité autorisant une occupation permanente n'avaient été réunies. Sa position aux portes de la ville du I ${ }^{\mathrm{er}} \mathrm{s}$. avant J.-C., offrant, comme de nos jours, un lieu d'expansion privilégié pour une cité comprimée dans un cadre resserré de collines, a sans doute suscité, assez tôt, l'apparition de constructions vraisemblablement disséminées comme le suggère l'éparpillement des traces laissées par les activités céramiques de l'époque augustéenne (Desbat et alii, 1994 : chap. V - Les origines du quartier).

Dès l'origine, le site devait être traversé par la Voie I, présente sous la forme d'une voie rurale bordée de fossés, reprise d'un possible chemin protohistorique (Chapotat. 1959, p. 1-2). Nous pouvons également retenir l'existence d'un chemin établi au sud de M9. parallèlement à la limite de cadastration. En effet, nous

116 Ce comblement est réalisé avant l'installation de l'état IA de la maison des Dieux Océans. 
observons, notamment sur la figure $39^{117}$, une rupture stratigraphique de part et d'autre de cet axe. Au nord, le terrain naturel limoneux (couche V 5 65) se présente à une altitude de $149,37 \mathrm{~m}$. tandis quau sud, il ne dépasse pas la cote $148.60 \mathrm{~m}$ et est recouvert par une couche composée de sable et de cailloutis (couche IX 28 42) qui pourrait correspondre à l'aménagement d'un chemin. D'autres passages de même nature devaient vraisemblablement quadriller l'ensemble de la plaine.

\section{LES RUES : AXES DE DÉVELOPPEMENT ET TÉMOINS DE L'ÉVOLUTION URBAINE}

\section{LE FAUBOURG SAINT-ROMAIN-EN-GAL}

Au cours de l'époque augustéenne. l'implantation humaine va se densifier le long de la Voie $\mathrm{I}^{1 / 8} \mathrm{et.}$ semble-t-il. le long de la Voie III. En effet, celle-ci pourrait être ancienne dans la mesure où elle est bordée de murs de façade en mortier blanc dont nous ne retrouvons pas les équivalents le long de la Voie II. Si la Voie I correspond au plus court chemin permettant de traverser la vallée, la Voie III. par sa position oblique. pourrait constituer une route médiane desservant les terrains de l'ancienne île du Rhône ainsi que l'amorce de la voic qui se dirigeait vers Lyon par la rive droite. Sa date d'apparition demeure toutefois à préciser par une étude approfondie du mobilier issu des fouilles de 1993.

A la fin de l'époque augustéenne, le faubourg SaintRomain-en-Gal poursuit son extension. Le développement des constructions le long des voies principales entraîne la mise en place d'un réseau complémentaire de rues secondaires destiné à faciliter l'accès à de nouvelles parcelles. Ainsi, la création de la Voic Il débute par l'édification de façades qui fixent les limites de terrains situés à l'onest de la Voie III. Les nouveaux murs. construits avec du mortier jaune, peuvent correspondre soit à une extension des édifices primitịss, soit à de nouveaux bâtiments qui viendraient s'adosser à ceux-ci. Comme dans le même temps, les parcelles de la maison aux Cinq Mosaïques (état IC) et des Petits Entrepôts s'avèrent très peu bâties, nous pouvons penser que la Voie II est alors une route transversale aménagée aussi bien pour accéder à de nouvelles constructions que pour viabiliser des terrains, dans un quartier que les Voies I et III ne suffisent plus à desservir ${ }^{119}$.

117 Une différence est également visible dans la maison des Dieux Océans. entre le sondage I 51, situé au nord de l'axe et le sondage I 66, situé au sud. En ce qui concerne le sondage Voie II 2. son exiguïté ne permet pas d'avoir de certitude quant à la nature de l'espace qui se développe au sud de M9. Toutefois, la tranchée oblique, par sa présence, nous incite à envisager, là aussi, l'existence possible d'un chemin (ef. p. 16).

118 Il est possible yue, près du fleuve, de part et d'autre de la Voic I. des îlots d'habitations soient déjà organanisés, formant ainsi le noyau d'un premier faubourg qui s'élendra le long des voies d'accès.

119 Cette hypothese est valable pour la portion de rue
L'empierrement simple de la première chaussée (couche 77), par ailleurs dépourvue de trottoir et de collecteur, nous incite à penser que nous avons bien affaire à une voie de desserte dans un secteur en cours d'aménagement. Sa faible durée de vie, dont témoigne son usure peu marquéé, semble correspondre à une étape préliminaire destinée à faciliter les aménagements des parcelles et permettant notamment la réalisation des constructions de l'état ID de la parcelle de la maison aux Cinq Mosaïques. Cette phase est ensuite suivie de l'établissement d'une nouvelle chaussée qui restera en service plus longtemps. Le fait que ce deuxième niveau de circulation soit bordé de rigoles sommaires aux dimensions modestes démontre que, jusqu'aux années 40, les eaux de ruissellement sont peu abondantes et, donc. que les parcelles limitrophes demeurent partiellement bâties et peu densément habitées.

\section{LE GUARTIER URBAIN DE SAINT-ROMAIN-EN-GAL}

Ce n'est qu'un peu plus tard, sous le règne de Claude, que l'importance de la Voie II se confirme avec l'installation d'un premier réseau d'adduction d'eau et d'un collecteur. La conception primitive de cet égout. destiné à la récupération des eaux usées en provenance des parcelles situées à l'ouest de la chaussée, est l'indice d'un aménagement concerté entre des particuliers et l'autorité publique. Nous sommes en présence d'une phase véritablement urbaine de développement qui voit le quartier s'étendre vers l'ouest ${ }^{12()}$ et se couvrir de constructions. Dorénavant, les jardins des grandes maisons constitueront les seuls espaces découverts.

Avec l'édification des Grands Entrepôts et du Bâtiment Commercial de la zone V. la Voie II acquiert un aspect commerçant qui ne se démentira plus. Ces constructions entraînent une augmentation de la circulation des véhicules qui constitue la source essentielle des dégradations et des réfections que vont subir les chaussées successives. La rue du Commerce prend réellement naissance à cette époque.

Il nous reste à déterminer si l'installation de l'égout public constitue un aménagement isolé ou s'il s'insère dans un programme plus systématique de drainage de l'ensemble des îlots. La question se pose en effet dans la mesure où les collecteurs des diverses rues présentent des morphologies variées el des chronologies diffé-

comprise entre la Voic I et la Voie III. Au nord de cette derniere, nous ne pouvons nous prononcer. en l'absence de fouilles, sur le prolongement de la Voie II qui est pressenti depuis les recherches géophysiques (Hesse e't alii, 1978). Nous ignorons tout de la date d'apparition de ce tronçon.

120) Celle constatation faite pour le secteur concerné par celte étude peut vraisemblablement s'appliquer, suivant d'autres directions, voire même sur l'ensemble de la plaine de lat rive droile. 
rentes 121. Létude à venir de la Voie III devrait nous permettre de savoir si nous retrouvons la trace d'une relation unilatérale entre l'égout et les constructions qui bordent l'un des côtés de la rue, identique à celle de la Voie II. Si une telle observation se renouvelait, la mise en place des collecteurs constituerait un programme urbain très structuré dans lequel chaque égout. obéissant par ailleurs à des règles précises d'implantation (cf. la position de l'égout dans l'état $2 \mathrm{~B}$ de la Voie I), ne desservirait qu'une moitié de rue.

Cependant. cette hypothèse nous conduit à envisager une organisation trop rigide qui ne sera pas respectée par la suite dans la mesure où nous observons des raccordements d'égouts domestiques provenant indifféremment d'un bord de la chaussée ou de l'autre. Néanmoins. sans tirer une règle nécessairement contraignante de l'exemple de l'égout de la Voie II. l'implantation de collecteurs comportant des débouchés pré-détinis peut néanmoins traduire l'état de développement du quartier a une époque où sont installées de vastes demeures qui ne sont dotées que de peu d'évacuations 122.

La mise en place, vers le début de l'époyuc flavienne. d'un second réseau d'adduction d'eau, alors que le premier paraît encore en service (ci. supra. p. 52), suggère une augmentation des besoins, peut-être liée à l'accroissement du nombre de bassins d'agrément dans les jardins. Toutefois. le nombre élevé de tuyaux. de même que leur concentration, soulèvent le problème de leurs provenances et de leurs destinations. Les canalisations étant les plus nombreuses à la hauteur des thermes des Lutteurs. deux hypothèses antagonistes sont envisageables.

Dans le premier cas. ces tuyaux. issus de différents captages réalisés sur la rive droite. convergeraient vers l'édifice thermal pour en assurer l'alimentation. Mais nous pouvons, dans ce cas. nous interroger sur les raisons qui les font contoumer le bâtiment par le nord. alors qu'il aurait été plus simple qu'ils pénètrent dans le bâtiment par l'ouest. du côté de la Voie I (fïg. 29).

Dans le second cas, au contraire. ces conduites partiraient du secteur thermal. ou des alentours. pour alimenter les différentes parties du quartier. Elles émaneraient alors d'un aqueduc qui pourrait être une ramification de l'un de ceux qui desservent la rive gauche de la ville. La finalité de cet ouvrage pouvant

121 l'égout de la Voie II est. semble-t-il, le plus ancien des égouts en service dans le quartier. Celui de la Voie I. en lout cas le tronçon qui longe l'îlot A. est plaqué contre le Portique Monumental dont la construction intervient au milicu du I ${ }^{e r}$ s. Le's dates de construction des atutes collecteurs restent à préciser.

122 Un exemple de ce type nous est fourni par la maison au Vivier yui est contemporaine des transformations de la Voic II (Savay-Guerray et alii. 1992, p. 731. Cette hatbitation de plus de $2200 \mathrm{~m}^{2}$. dont l'entrée est situce a l'est. ne posside qu'un égout domestique qui la traverse sur une grande distance pour se diriger vers la ruc qui longe sa façade orientate. être l'approvisionnement des thermes, il conviendrait alors d'envisager la présence d'un pont aqueduc en face du site.

N'ayant aucune trace de cette construction, nous ne pouvons pour l'instant favoriser l'une ou l'autre de ces solutions. Cette constatation laisse une nouvelle fois en suspens le problème de l'alimentation en eau des quartiers de la rive droite. Il nous reste à espérer que la fin du dégagement des thermes des Lutteurs nous fournisse des éclaircissements sur la destination (ou le point de départ) de cette batterie de tuyaux.

Less deux réseaux d'adduction d'eau mettent en relief la place importante prise par les canalisations en bois dans le système de distribution. S'il n'apparaît. jusqu'à présent. aucune antériorité dans l'utilisation du bois ou du plomb ${ }^{23}$. leur emploi s'avère complémentaire. En effet. le bois, d'un usage moins souple, semble réservé aux conduites principales du résea qui amènent l'eau du point de captage au secteur a desservir. tandis que le plomb apparât des que l'on a affaire aux luyaux sur lesquels sont effectués les branchements locatux. notamment ceux des particuliers. Il pourrait en être ainsi pour chacun des réseaux des différentes époques ${ }^{12 t}$.

\section{LE CENTRE URBAIN \\ DE SAINT-ROMAIN-EN-GAL}

Au cours du IICs. après J.-C.. l'installation d'un premier dallage de la chaussée traduit une rupture dans l'évolution de la rue et entraîne une modification de l'aspect du quartier qui semble être une conséquence de la construction, dans le courant du $]^{\mathrm{er}}$ s., du Complexe Monumental de la rive droite (Savay-Guerraz. Prisset. 1992). Celui-ci peut être considéré comme un prolongement de l'axe monumental de la rive gatuche qui comprend notamment le fortum et le théâtre. Dès lors. la ville se divise en quatre grands quartiers répartis de part et d'autre des deux axes perpendiculaires constitués par le fleuve et les ensembles monumentaux des deux rives. Si cette organisation montre bien l'ampleur prise par la ville, elle introduit aussi un éclatement du cour urbain. même si le Complexe Monumental de Saint-Romainen-Gal joue un rôle fédérateur. Dans ces conditions. nous pouvons considérer que le quartier, tout du moins sa partie sud, fait dès lors partie du centre étendu de Vienne. Cette nouvelle place au sein de la ville va accrôttre son importance é élever son statut.

Tout en constituant une amélioration des conditions de circulation. le dallage des rues principales est une nouvelle expression de la structuration urbaine. Désor-

123 Les cantilisations des deux yypes coexistent des la mise en place du premier résealu.

124 Dans la Voic III. des conduites en bois se rencontrent sur pas moins de quatre niveaux. Cetle rue chant en cours d'élude, il n'est pas ancore possible de préciser l'insertion de és canalisations dans les réscaux déjà reconnus. 
mais, les quartiers exra muros ${ }^{125}$ participent directement, par le biais de leur infrastructure, à la décoration de la ville, à sa valorisation. ainsi qu'à sa monumentalisation.

Vers la fin du IIC s.. le stade ultime de l'aménagememenl est alteint avec l'implantation systématique de trottoirs dans les rues principales. Nous sommes en présence de véritables constructions, composées d'une fondation maçonnée recouverte de dalles calcaires, qui s'avèrent bien différentes des trottoirs bétonnés ou de terre battue qui se rencontrent dans les villes campaniennes. A Pompéi, par exemple, ces derniers offrent des largeurs variables et sont présents. même s'il ne s'agit que d'une bordure de chaussée. sur les deux côtés de la rue. A Saint-Romain-en-Gal, au contraire, les largeurs des trottoirs sont homogènes ${ }^{26}$ et les rues n'en reçoivent qu'un seul. Sur les Voies II et III, nous observons que leur implantation s'est effectuée le long de la façade ouest, ce qui semble signifier que les accès aux bâtiments des îlots $\mathrm{A}$ et $\mathrm{C}$ étaient essentiellement situés de ce côté-là, si nous excluons les accès aux grandes demeures qui sont placés sur la Voie I.

L'ajout de ces maçonneries engendre un fort rétrécissement de la zone de roulement. Dès lors, la chaussée n'occupe plus que les deux tiers de la largeur de la rue. Cette réduction systématique correspond peut-être à une diminution du trafïc des véhicules dans le quartier. Néanmoins, il nous reste à valider cette hypothèse dans la mesure où les nouvelles voies offrent encore suffisamment de place pour que deux véhicules puissent se croiser sans difficultés.

Créés de toutes pièces (Voies III et X) ou reprenant des cheminements antérieurs ${ }^{127}$, les trottoirs viennent en complément des portiques privés ${ }^{128}$ et concourent à la réalisation d'une circulation pićtonne généralisée et cohérente. Cette prise en compte du confort du passant et l'aspect monumental de ces trottoirs parachèvent la mise en valeur de la ville, en remodelant la physio-

125 Les fouilles de la place Camille-Jouffray. sur la rive gauche, ont mis en évidence le même phénomène pour le quartice sud (Baratle ef alii, 1990), p. 17).

126 I.es dalles ayant pratiquement toutes disparues, nous ne pouvons comparer que les largeurs des fondations. Cellesci sont de l'ordre de $1.40 \mathrm{~m}$ à $1.50 \mathrm{~m}$ dans les Voies III el X. Le trottoir de la Voie $I 1$ mesure $1.75 \mathrm{~m}$ dans sa partic pleine et $1.90 \mathrm{~m}$ à lat haukeur du drain. Cette largecur plus importante pourrait être l'indice d'une plus grande circulation piétonne dans cette rue. Mais il ne s'agit là que d'une simple hypothèse.

127 Dans la Voie II, ke trottoir se superpese au bals-côté de $1.60 \mathrm{~m}$ de largeur mis en évidence à l'état 2C de la rue. Celuici ne faisait sans doute l'objet d'aucun aménagement spécificue, à limage de la bande de terrain yui se rencontre au sud de la Voie I. entre les exedres du Portique Monumental (lig. 2). Il convient de noter qu'aucun trottoir. antérieur à ceux du dernier étal, n'a jusqu'à présent élé mis en évidence dans les niveaux anciens des différentes rues.

128 Au cours des époques précédentes. les portiques sont aménagés au détriment des parcelles. nomie du quartier. Celui-ci a atteint son apogée et. dès lors, ne connaîtra plus de transformations de grande ampleur.

Cet aménagement est accompagné d'un dernier exhaussement des chaussées dont la raison d'être demeure encore inconnue. Il s'agit d'un travail considérable qui nécessite, non seulement, d'apporter des remblais, comme au cours des changements de niveau antérieurs, mais surtout de déplacer chaque dalle et ceci sur l'ensemble des voies principales. Nous imaginons aisément les conditions de circulation durant la période d'exécution. Ces chaussées perdureront telles quelles jusqu'à l'abandon du site.

A l'issue de cette évolution, nous pouvons remarquer quaucune des trois voies principales actuellement dégagées ne se superpose à un axe de la cadastration initiale. Cette constatation nous amène à penser que les chemins basés sur les cardines des centuries n'étaient pas, compte-tenu de la courbure de fleuve. les plus aptes à assurer une desserte satisfaisante de cette partie de la vallée. Le découpage interne des centuries a toutefois gardé une influence certaine en ce qui concerne les limites parcellaires ainsi que dans l'établissement des rues secondaires du site (voies du secteur nord-est et passages de l'îlot A).

\section{LA PARCELLE DE LA MAISON AUX CING MOSAÏQUES AU SEIN DE L'TLOT A}

Dans ce contexte général, la mise en correspondance des mutations qui ont affecté les parcelles occupées par la maison aux Cing Mosaïques et la maison des Dieux Océans permet de cerner l'évolution, non pas du quartier de Saint-Romain-en-Gal, mais de l'un de ses secteurs (tabl. XVII). En effet, cette distinction doit être faite dans la mesure où le développement s'opérant. semblet-il, par phases de croissance successives, l'îlot A n'est pas nécessairement représentatif. à chaque période, de l'occupation humaine sur l'ensemble de la plaine.

\section{DES ORIGINES À L'ÉPOgUE DE CLAUdE}

Alors que lat partic sud de l'îlot accueille très tôt des bâtiments de grande taille. la partie nord reste assez. longtemps vierge, ne révélant que des édicules. En l'absence d'aménagements d'ampleur. les traces de l'organisation spatiale existant dans la partic nord de l'îlot avant la construction de la maison au Grand Péristyle et du Bâtiment Commercial sont ténues, mais néanmoins suggestives. Notre but. dans cette partic, n'est pas d'analyser en détail l'agencement des quelques structures retrouvées (édicules. puits, fossess) mais de fournir des indices qui permettent de mieux cerner l'environnement de cette période ${ }^{29}$.

129 Les résultats fournis par les fouilles de la maison au Cing Mosaïques et de la Voie 11 montrent qu'il serait nécessatire de reprendre l'examen des niveaux anciens de l'ensemble de l'îlor. 
Tabl. XVII - Evolution de l'îlot A.

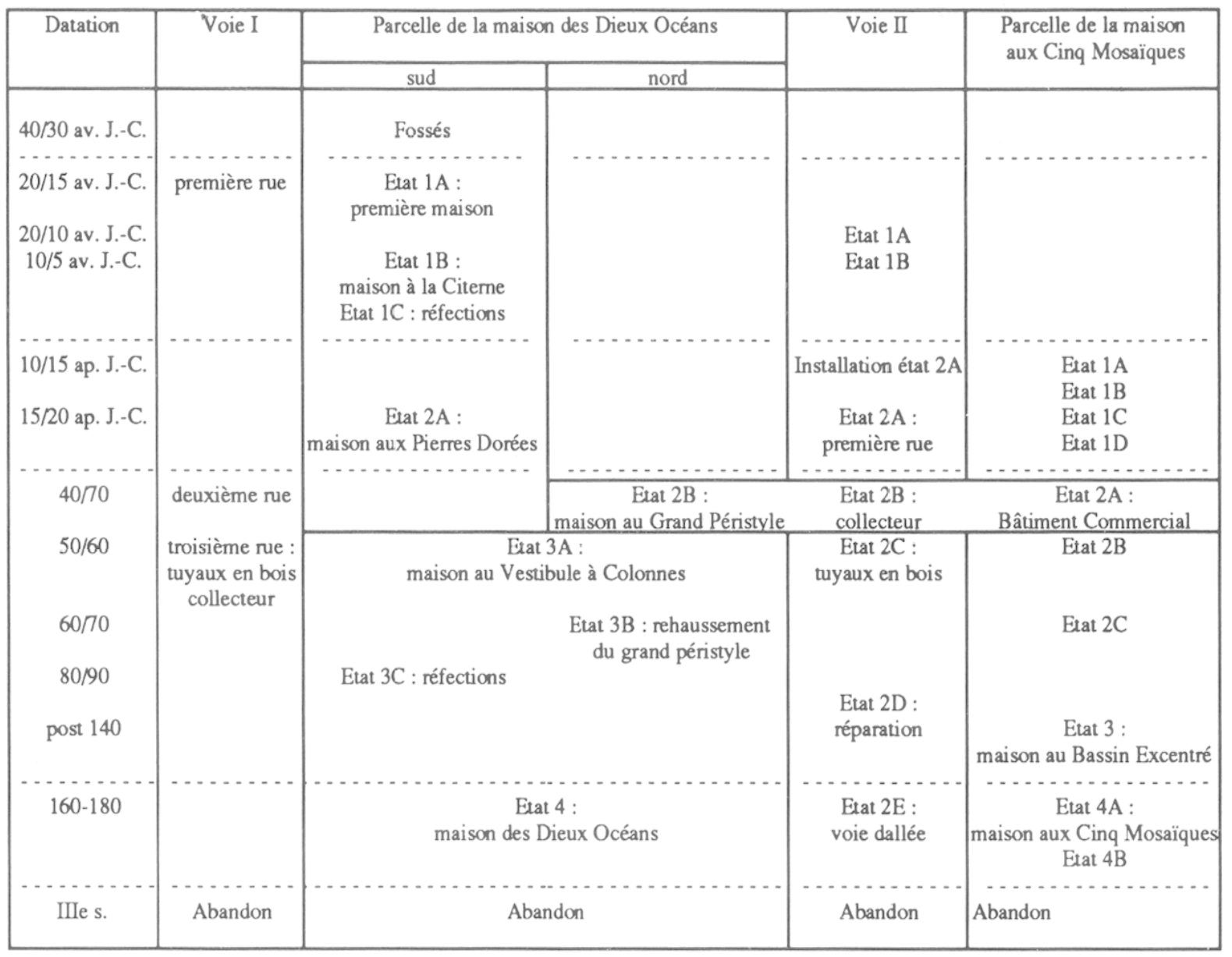

Ainsi que nous l'avions déjà signalé (Desbat ét alii. 1994 : chap. III - L’état 2B. Le plan), un rapport proche de 2/1 transparaît dans la structure de cette demeure (tabl. XVIII). Aucun des ensembles déterminés ne se présente sous une forme rectangulaire régulière. Indépendant de l'orientation de la Voie II. ce gauchissement ainsi que les fluctuations des largeurs suggèrent qu'un laps de temps assez. long s'est écoulé entre le moment où l'unité spatiale initiale, que nous pouvons supposer régulière, a été définie et la date de construction de la maison.

Cette unité semble être constituée par une jugère (240 pieds x 120 pieds) flanquée, au sud ou au nord, d'une bande de terrain est-ouest dont la largeur doit être comprise entre 6 pieds $(1 / 20$ d'actus) et 10 pieds. Cet élargissement peut correspondre à l'emprise d'un chemin dont l'emplacement n'a été mis en évidence à aucune des extrémités de cette jugère. soit parce qu'il n'a jamais été précisément matérialisé. soit parce que sa trace s'avère trop ténue ${ }^{130}$. Ainsi, la présence d'une construction. accolée au mur limite nord des états 1 et $2 \mathrm{~A}$ de la mai-

130 Les passages des Grands Entrepôts è du Bâtiment Commercial constitueraient, dans l'esprit, si ce n'est en emplacement, des résurgences de ces dessertes. son des Dieux Océans (Desbat et alii. 1994 : chap. III - l'état 2A, Le contexte urbain), montre que cette circulation, si elle se trouvait sur le flanc sud, dut disparaître assez tôt.

A quelle époque faut-il faire remonter l'apparition de ce découpage? Cette jugère apparaissant limitée, à l'est par la Voie II et, à l'ouest, par la rue sur laquelle ouvre la maison au Grand Péristyle, il est tentant d'établir une coïncidence de créations. Mais, cette partition a pu tout aussi bien être définie lors de l'établissement du cadastre dans la mesure où l'implantation de ces deux axes de circulation semble s'appuyer sur des limites de propriétés créées antérieurement. Compte-tenu des dimensions de l'unité parcellaire et du gauchissement des formes, la seconde hypothèse paraît la plus probable. Cependant, seule une étude plus précise du positionnement des centuries permettra de confirmer cette genèse.

La disposition de la partie ouest de la maison au Grand Péristyle (tabl. XVIII, ensembles C et D) et la présence supposée de deux parcelles à l'emplacement du Bâtiment Commercial (cf. supra, p. 44) nous amènent à proposer une division de la jugère en quatre parcelles de 120 pieds par 60 pieds, suivant une orientation estouest. 
Tabl. XVIII - Modules sur lesquels s'appuic l'organisation de la maison au Grand Péristyle.

\begin{tabular}{|c|c|c|c|c|}
\hline Ensembles & $\begin{array}{c}\text { Largeur } \\
(\mathrm{m})\end{array}$ & $\begin{array}{l}\text { Largeur } \\
\text { (pied) }\end{array}$ & \begin{tabular}{|c|}
$\begin{array}{c}\text { Longueur } \\
(\mathrm{m})\end{array}$ \\
\end{tabular} & $\begin{array}{l}\text { Longueur } \\
\text { (pied) }\end{array}$ \\
\hline $\begin{array}{l}\text { A - Bâtiment Commcrcial } \\
\text { + portique ouest du jardin de la maison } \\
\text { + passage du Bâtiment Commercial }\end{array}$ & 17,75 & 60 & 37,9 & 128 \\
\hline B - jardin et portique nord du jardin & 18,55 & 63 & 37,6 & 127 \\
\hline $\mathrm{C}$ - maison et portique ouest du jardin & 17,0 & 57,5 & $?$ & ? \\
\hline $\begin{array}{l}\text { D - parcelle bordée par la maison au } \\
\text { Grand Péristyle }\end{array}$ & 20,0 & 67,5 & ? & ? \\
\hline
\end{tabular}

Une confirmation de ce découpage nous est peut-être fournie par l'égout de vidange M48. En effet. nous remarquons que son tracé suit grossièrement la limite des deux parcelles orientales. La position de cet égout ne répondant pas à des critères évidents d'implantation par rapport au bassin qu'il draine, il est possible que le choix de son emplacement soit une conséquence de l'organisation parcellaire primitive - l'égout reprenant. par exemple. le tracé d'un fossé de bordure. Si tel était le cas, la bande de terrain supplémentaire se situerait alors sur le côté nord (fig. 1(06).

La mise en place de cette subdivision n'est pas nécessairement contemporaine de la création de la jugère. Celle-ci, en tant que telle. représente un lot de faible importance si nous prenons comme référence les dotations effectuées à des colons qui s'échelonnent de deux à une centaine de jugères (Chouquer. Favory. 1992. p. 39). Dans ces conditions, la partition peut très bien n'intervenir que dans un second temps, atu moment où les constructions commencent à investir la plaine. ou lors de la création de la Voie II.

\section{LE BÂTIMENT COMMERCIAL AU SEIN DE LA MAISON AU GRAND PÉRISTYLE}

Lors de l'étude de la maison des Dieux Océans, nous avions souligné la simultanéité de construction de la maison au Grand Péristyle el du Bâtiment Commercial (Desbat ét alii, 1994 : chap. III - L'état 2B : Les élévattions de l'état 3A). Si nous replaçons ces deux ensembles dans leurs propres contextes évolutifs, nous pouvons affirmer qu'il s'agit bien d'une structure unique. cohérente dans le temps.

Occupant trois des quatre parcelles définies antérieurement, cette domuss, dont le corps de logis nous demeure inconnu, fournit des renseignements importants sur le développement d'une habitation à l'intérieur d'un îlot. Deux aspects retiennent surtout notre attention : la disposition des accès et l'ampleur du péristyle.

D'après l'organisation générale de la parcelle. l'entrée principale de la maison au Grand Péristyle doit déboucher à l'ouest, sur une rue parallèle à la rue du Commerce (fig. 107). Cette demeure traversant tout l'îlot, la présence du passage du Bâtiment Commercial nous montre qu'un débouché sur la rue du Commerce a également été prévu lors de la construction. Aboutissant directement au péristyle, ce passage. large de $1,70 \mathrm{~m}$. est clos à chaque extrémité par un mur conservé seulement en fondation. au-dessous du niveau général de circulation, qui se situe à $151,20 \mathrm{~m}$ dans la rue du Commerce et à $151.00 \mathrm{~m}$ dans la maison au Grand Péristyle. Cette disposition se retrouvant dans le passage des Grands Entrepôts, ces maçonneries nous indiquent l'emplacement de portes et soulignent le caractère privatif de ce passage.

Par ailleurs, l'existence du passage du Bâtiment

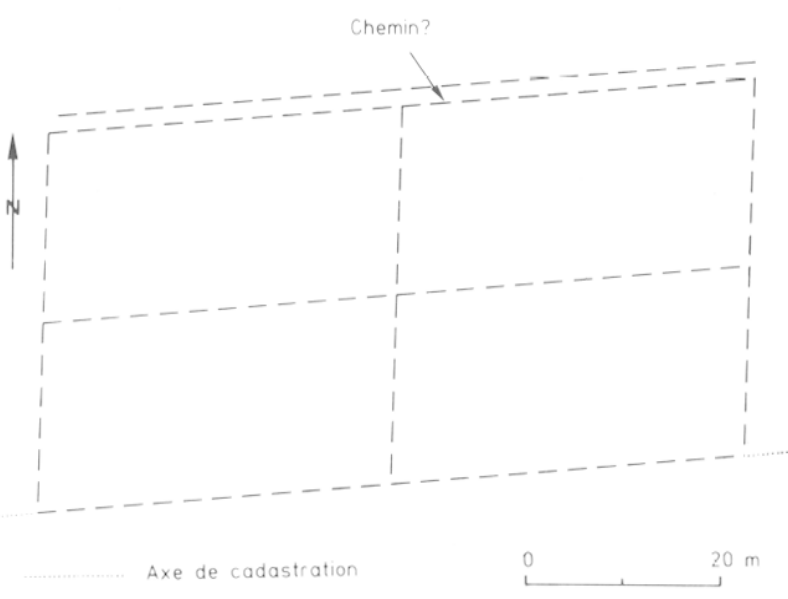

Fïg. 106 - Organisation parcellaire des terrains à l'emplacement de la maison au Grand Péristyle è du Bâtiment Commercial.

Commercial et sa situation au sud de la parcelle nous précisent que le passage des Grands Entrepôts n'est pas rattaché à la maison au Grand Péristyle et qu'il ne constitue pas davantage une voie publique. Il serait en effet étonnant que la maison possède en propre un accès sur la Voie II alors qu'une ouverture sur la Voie IV pouvait lui assurer le même service. De ce fait, il nous est permis de penser que ce passage, dotés de portes et aménagé au moment de la construction des Grands Entrepôts. constitue également l'accè̀s annexe, privé. d'une parcelle située à l'ouest du bâtiment des Grands Entrepôts ${ }^{131}$. Cet agencement, répondant à celui de la

1.31 Il est d'ailleurs vraisemblable que ke passage des Grandi lintrepôts el les pièces qui ouvrent sur lui n'ont pas de rapport direct avec les Grands Entrepôts, proprement dits. Ceux-ci, identiques en conception el construits à la même époque que les docks de la rive gauche (Le Bot. 1989). constituent à n'en pas douter un édifice public sans doute destiné au stockage de denrées el peut-être réservé aux besoins du quarticr. 


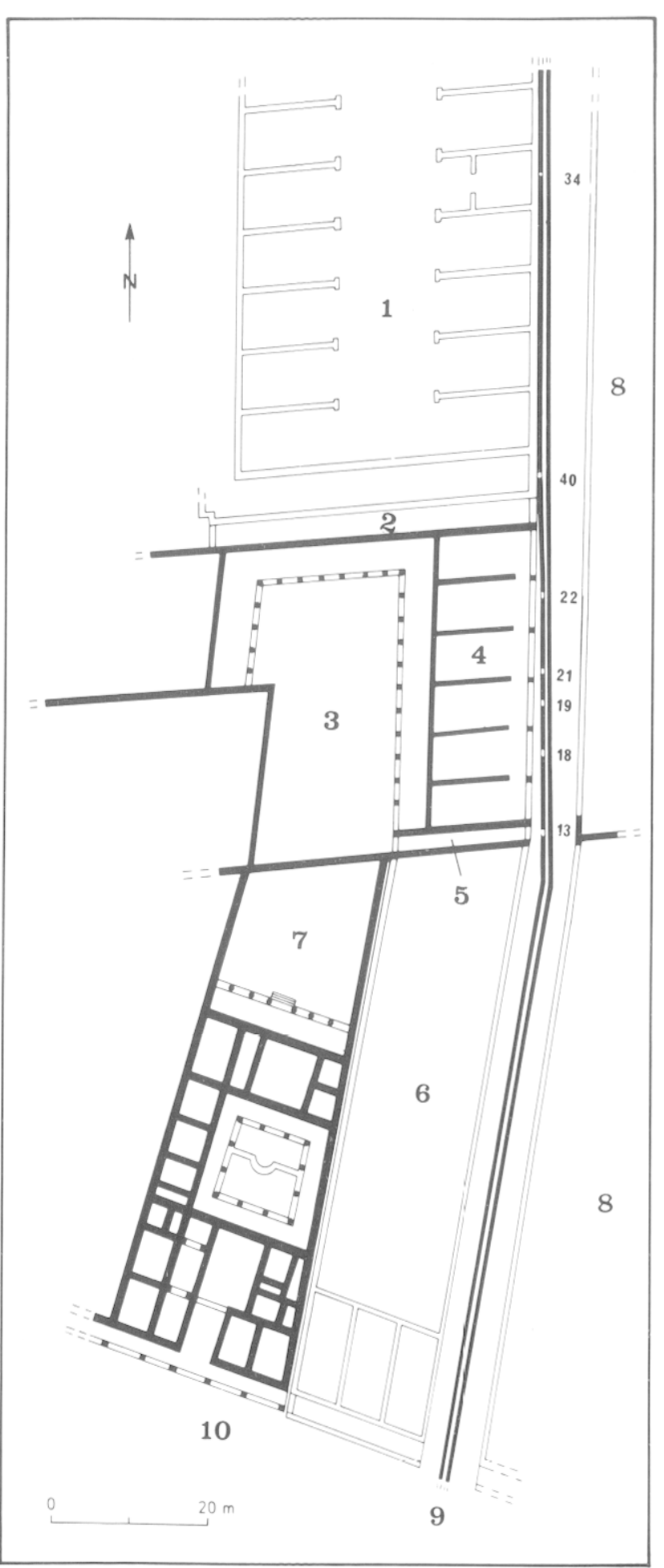

Fig. 107 - Plan de l'îlot A au moment de la création de l'égout de la Voic II, au milicu du I ${ }^{\mathrm{er}} \mathrm{s}$. après J.-C. : 1, Grands Entrepôts : 2, passage des Grands Entrepôts : 3, maison au Grand Péristyle : 4, Bâtiment Commercial (état 2A): 5. passage du Bâtiment Commercial : 6. parcelle des Petits Entrepôts (petit bâtiment commercial au sud, construction indéterminéc au nord) : 7, maison aux Pierres Dorées : 8. îlol C: 9. égout public de la rue du Commerce, avec les débouchés prévus au moment de sa construction : 10, Voic I. maison. traduit la nécessité pratique de posséder un débouché sur chaque rue. montrant ainsi que les îlots $A$ et $B$ ne forment en réalité qu'un unique el vaste ensemble, comparable à l'îlot C.

Nous pouvons estimer la superlicic lotale de la maison au Grand Péristyle aux alentours de $197.5 \mathrm{~m}^{2}$. Le portique et le jardin couvrent $910 \mathrm{~m}^{2}$ (soit $46 \%$ de cette surface) tandis que le Bâtiment Commercial et le passage contigu occupent $511 \mathrm{~m}^{2}(26 \%)$, ce qui ne laisse au corps d'habitation que $5.54 \mathrm{~m}^{2}(28 \%)^{1.32}$.

L'organisation interne de la parcelle confirme la faible part laissée à l'habitat proprement dit. Celui-ci se trouve en effet relégué à l'ouest, dans la partie la plus étroite et la plus confinée, alors qu'il aurait pu bénéficier d'une vaste façade sur la Voie II 13.3. Dans ces condi-tions, il ne fait aucun doute que le propriétaire a voulu mettre en valeur le secteur commercial. Toutefois, il n'a pas lotalement négligé son confort si l'on considère l'importante emprise du péristyle.

Au sein de cet espace. la fouille de la maison aux Cinq Mosaïques, en nous révélant l'existence. dès cette époque, d'un égout issu du grand péristyle, nous assure de la présence d'un bassin dont la forme et l'emplacement demeurent inconnus. La forme générale du poitique, notamment du côté ouest, et son évolution ultérieure suggèrent déjà l'existence d'un bassin en U. Cependant. aucun indice pouvant préciser l'existence d'un tel aménagement n'a été fournit par le sondage I 65. situé à l'emplacement de l'angle saillant du jardin 134

L'absence d'évacuation à l'extrémité ouest du passage du Bâtiment Commercial (tandis qu'il existe, à l'autre extrémité. un débouché dans le collecteur de la Voie II (tabl. I) et le choix de l'emplacement de l'évacuation semblent signifier que ce bassin ne devait guère se poursuivre au sud de son orifice de vidangel 35 . Peut-être était-il cantonné en face du bâtiment d'habitation. sur le

132 Cetle valcur englobant un jardin de dimensions indéterminées, mis en évidence par une peinture murale (Desbat ét alii. 1994 : chap. III - L'état 2B. Les peintures). les pièces d'habitation occupent. en réalité. une superficie encore plus réduite.

133 Celte structuration de la maison permet d'envisager une possible permanence du lieu d'implantation de l'habitat. Ainsi. le corps de logis pourrait, soit être plus ancien. soit reprendre l'emplacement d'une habitation qui aurait élé établie dans la seule parcelle nord-ouest. avant que la fusion des trois parcelles n'ait été réalisée. Cette hypothèse. qui reste bien entendu à vérifier. laisse présager une évolution asse\% complexe de ce secteur occidental.

1.34 les autres sondages, susceptibles d'apporter des renseignements sur cette construction. noont pas été conduits assez profondément.

135 Les orifices des conduits de vidange sont généralement situés aux angles ou aux extrémités des bassins. voire dans les exedres. 
seul côté est, s'il s'agit d'un bassin rectangulaire, ou sur trois côtés dans le cas d'un bassin en U. Ce dernier pourrait ne pas occuper toute la longueur du côté ouest. laissant ainsi un accès au jardin. ce qui expliquerait l'absence de traces en I 65 (un tel dispositif se retrouvera aux deux exirémilés du bassin en $\mathrm{U}$ de la maison au Vestibule à Colonnes).

Si nous retenons l'idée que la pièce d'eau, quelle que soit sa forme. n'est présente qu'en face du corps de logis, il nous faut envisager la possibilité que l'espace central du péristyle ne constitue pas un ensemble homogène. Si la partie nord peut se concevoir comme un jardin. la partie sud, avec un accès charretier possible ${ }^{136}$, peut avoir servi de cour de service, la branche est du portique assurant la desserte générale des lieux. Il convient de noter que cette branche n'ouvre pas sur le passage du Bâtiment Commercial. Elle en est séparée par un mur en élévation qui nous indique clairement que ce passage ne constitue pas l'issue naturelle du portique mais bien celle de l'espace central dans lequel il faut pénétrer pour pouvoir l'emprunter.

\section{LE BÂTIMENT COMMERCIAL AU SEIN DE LA MAISON AU VESTIBULE À COLONNES}

L'implantation privilégiée du Bâtiment Commercial semble indiquer que le propriétaire des lieux était un commerçant qui dût s'enrichir assez vite si l'on en juge par les acquisitions de terrains qui vont se succéder au milieu du Ier $^{2}$.

Tout d'abord, le passage du Bâtiment Commercial est réduit à un simple ambitus du fait d'une extension vers le nord de la parcelle des Petits Entrepôts. Cette transformation n'ayant pu s'accomplir qu'avec l'autorisation du propriétaire de la maison au Grand Péristyle, il est pratiquement certain, compte-tenu des transformations qui vont se succéder, que celui-ci est lui-même à l'origine de cette suppression.

En effet, peu de temps après la construction des deux bâtiments septentrionaux des Petits Entrepôts. la maison au Vestibule à Colonnes est à son tour bâtie (fig. 108). La partie sud de cette domus, que nous considérons comme un agrandissement de la maison au Grand Péristyle devient, d'une certaine manière un nouveau débouché du noyau initial. La conjonction et la cohérence de ces événements nous conduisent à y voir l'œuvre d'un seul homme. Cette hypothèse, déjà formulée lors de l'étude de la maison des Dieux Océans, se trouve confortée.

Nous pourrions même envisager que l'inachèvement du Bâtiment Commercial, suggéré par les débouchés inutilisés du collecteur de la Voie II, soit lié à la création inopinée des Petits Entrepôts. Ainsi, de nouvelles possibilités d'extension apparaissant, le propriétaire

136 Avec $1,70 \mathrm{~m}$ de large, ce passage avait toutefois du mal à recevoir des charrettes possédant un écartement de roues de $1,50 \mathrm{~m}$ (cf. supra, p. 31).



Гig. 108 - Plan de la maison au Vestibule à Colonnes (état $3 \AA$ de la maison des Dieux Océans) avec le Bâtiment Commercial (ćtat 2B).

aurait retardé l'exécution des travaux dans l'attente d'une redéfinition de l'organisation de cette partie de l'îlot. Cependant, cette hypothèse est trop circonscrite à la maison au Grand Péristyle pour expliquer que les débouchés implantés devant les Grands Entrepôts soient restés, eux aussi, en attente de raccordement. Il faut plutôt prendre en considération l'apparition d'une contrainte qui touche l'îlot dans sa totalité ou l'ensemble du quartier. La cause la plus tangible serait liée au rehaussement général qui s'amorce à cette époque et qui paraît être en relation avec la construction de l'ensemble monumental.

Quoi qu'il en soit, la deuxième phase du Bâtiment Commercial est réalisée avant que les enduits qui couvrent le grand péristyle voisin ne soient appliqués. Cette réfection peut ainsi intervenir au moment de la construction des Petits Entrepôts.

Un autre témoignage de la cohérence des transformations nous est fourni par le maintien en service du premier réseau d'adduction d'eau. Nous avons formulé cette hypothèse pour la canalisation présente 
dans le portique du Bâtiment Commercial. Dans le nouveau contexte, il est tout à fait possible qu'elle ait continué à alimenter le bassin du grand péristyle, tout en pouvant répondre aux besoins des différentes cellules.

Mettant un terme à l'état 2B. un incendie entrâne là fin de cette structure commerciale et son rattachement probable à la maison au Vestibule à Colonnes (fig. 109). La mise en relation du bâtiment avec la maison. dont il devient une aile de service. constitue une indication claire de son appartenance à un même proprićtaire, ou plutôt à une même famille, si l'on en juge par la longévité de cet ensemble. En effet, près d'un siècle s'écoule entre sa construction et sa transformation en habitation.

L'incendie provoque la disparition des enduits à l'intérieur des boutiques. Or, nous observons la même absence d'enduits muraux dans le grand péristyle et l'aile ouest de la maison. Il est tentant de relier les deux événements dans la mesure oü. l'ex-Bâtiment Commercial étant au moins partiellement reconstruit, une période de travaux touchant le nord de la maison au Vestibule à Colonnes a pu débuter et ne pas trouver de conclusion avant que ne soit décidé le rehaussement complet de cette partie de la demeure.

L'utilisation du corps de bâtiment dans sa nouvelle affectation va durer une cinquantaine d'années. Durant cette période, le grand péristyle de la maison devait présenter une différence de niveau qui n'a pu disparaître qu'avec l'avènement de la maison au Bassin Excentré, au milieu du $\mathrm{II}^{\mathrm{s}} \mathrm{s}$. Or. dans l'état actuel des recherches, le remblaiement du deuxième jardin est daté de la seconde moitić du I"'s. Nous observons ainsi un décalage chronologique non négligeable qui nécessite le réexamen du mobilier provenant des remblais utilisés pour cette opération. Notons que la date de construction de la maison au Bassin Excentré est donnée grâce à une monnaie d'Hadrien qui fait fïgure d'exception dans un lot de matériel plutôt daté de la fin du I ${ }^{\mathrm{er}}$ ou du début du II's. (cf. supra, p. 102).

Il ne faut pas trop s'étonner de retrouver à cette époque des remblais issus de dépôts plus anciens. En effet, le site de Saint-Romain-en-Gal dans son ensemble a fait l'objet d'un rehaussement conséquent au cours du far $s$. Dans un tel contexte, toute nouvelle construction entame les terres précédemment déposées, qui fournissent ainsi des déblais réutilisables pour d'autres aménagements. Cependant, afin de préciser les faciès

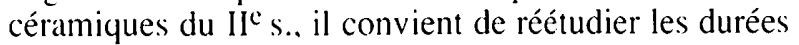
d'utilisation du mobilier céramique, ainsi que les dates d'apparition de nouvelles formes, notamment en ce qui concerne la céramique sigillée.

\section{LA MAISON AU BASSIN EXCENTRÉ ET LA MAISON AUX CINg MOSAÏgUES}

Avec la transformation du bâtiment en maison, fautil envisager la création d'une habitation locative ou un transfert de propriété ? Le maintien, après rehaussement, du passage de l'évacuation du bassin voisin pourrait

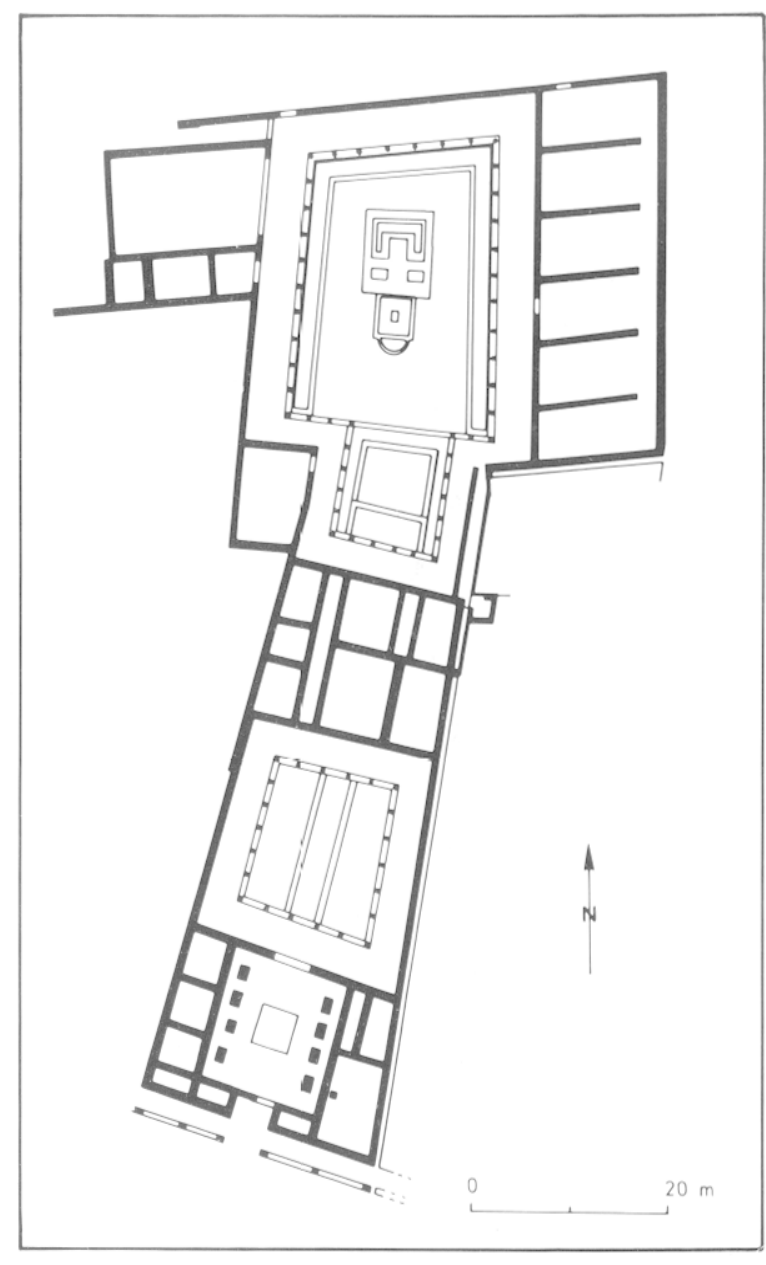

Fig. 109 - Plan de la maison au Vestibule à Colonnes (ćtat $3 \mathrm{~B}$ de la maison des Dieux Océans) lorsqu'elle intègre le Bâtiment Commercial (état 2C).

nous inciter à privilégier la première hypothèse. Toutefois, la relative importance de la superficie de la maison et surtout la structuration marquée de son organisation interne nous poussent à la considérer plutôt comme une demeure indépendante que comme une domus de rapport. De plus. si nous admettons l'existence d'une boutique de modestes dimensions, son caractère unique et sa parfaite intégration dans le bâtiment confortent la cohérence de l'ensemble et suggèrent que nous sommes en présence de l'habitation d'un nouveau commerçant désireux de s'installer dans le quartier.

Avec la construction de la maison aux Cinq Mosaïques, la rupture avec la demeure voisine est alors nettement consommée. Non seulement la canalisation M27, véritable cordon ombilical (fig. 110), est désormais coupée mais la mise en service d'un nouvel égout dans l'aile ouest de la maison des Dieux Océans. M602, provoque la création d'un nouveau cheminement qui emprunte la Voie IV pour venir se greffer sur le collecteur public de la Voie II (fig. 69).

Même si nous ne pouvons déterminer qui fut l'aménageur de la maison aux Cinq Mosaïques (un 


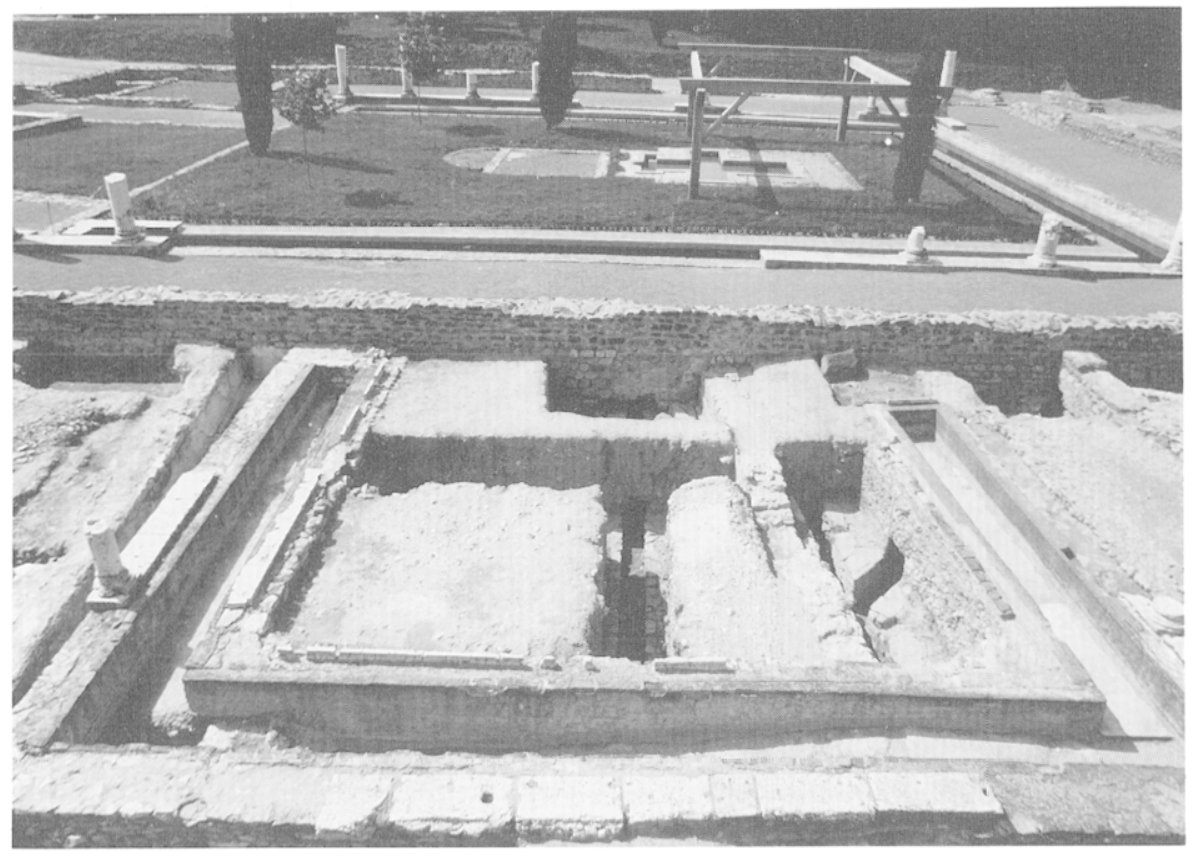

Fïg. $110-$

Au centre du jardin de la maison aux Cinq Mosaïgues. l'égout M27, évacuation du bassin en $\mathrm{U}$ du grand péristyle de la maison des Dieux Océans (ai l'arrière plan). symbolise le lien entre les deux parcelles tout au long de leurs histoires. descendant du propriétaire de la maison au Bassin Excentré ou un nouvel habitant ?), il convient de noter l'embellissement marqué de cette habitation. A plus d'un siècle de distance, nous retrouvons l'évolution de la maison au Grand Péristyle. Certes, la place disponible et le type de maison ne sont plus les mêmes. Au Ier $\mathrm{s}$.. les demeures sont plus majestueuses et plus expansives car elles se développent sur des regroupements de parcelles tandis qu'au Il゙ s., les nouvelles constructions n'ont a leur disposition que des terrains issus des scissions qui s'operent dans certaines des plus importantes demeures 1.37 .

Ces maisons sont-elles simplement devenues trop grandes pour leurs utilisateurs? S'agit-il d'une transformation de mentalité - un rejet du gigantisme - ou des effets d'une récession qui touche certains propriétaires ? L'état des recherches actuelles ne permet pas de formuler des réponses définitives mais de dégager des tendances. Dès le II $^{\mathrm{s}}$ s. nous observons des changements importants dans l'aspect et la structure des maisons : création systématique de secteurs thermaux. modifications des jardins, réduction des bassins en profondeur et en longueur (Savay-Guerraz. Prisset, Delaval, 1993, p. 91-92).

La maison des Dieux Océans semblait échapper à ces phénomènes. Nous savons maintenant qu'il n'en est rien. En effet. l'obstruction de l'égout de déversement du bassin en U. accompagnée par le fait qu'aucune nouvelle évacuation ne lui fut substituée. exclut toute possibilité

1.37 En dehors de la maison au Vestibule à Colonnes, la maison à la Grande Galerie présente également une amputation importante puisqu'clle sera coupée en deux. perdant la plus grande partic de son jardin qui sera dès lors occupé par un nouveau bâtiment indépendant (Savay-Guerraz ct alii, 199', p. 76-77). de vidange. Cette situation ne peut qu'entraîner la formation rapide d'un étang d'eau croupie. Ce nouvel état. peu plaisant et peu salubre. ne devant pas particulièrement être recherché, il nous faut plutôt envisager une suppression, pure et simple, de la pièce d'eau. Cette obstruction a pu intervenir durant l'existence de la maison au Bassin Excentré. et a eu pour conséquence de permettre le bouchage de l'égout lors de la construction de la maison aux Cing Mosaïques. Un nombre conséquent de fragments de colonnes ayant été retrouvé dans le comblement du bassin en $U$. il apparaît qu'une partic du portique (les branches est et nord ?) a été supprimée à cette occasion (fig. 71$)^{1.38}$.

Cette récession des bassins se retrouve également entre la maison au Bassin Excentré et la maison aux Cing Mosaïques. Le surface du plan d'eau passe de $15.1 \mathrm{~m}^{2}$ à $22.9 \mathrm{~m}^{2}$ tandis que le volume d'eau utilisé régresse de $10.1 \mathrm{~m}^{3}$ a $9.5 \mathrm{~m}^{3}$. la profondeur du nouveau bassin étant moindre. Ainsi, malgré l'augmentation de superficie, la quantité d'eau consommée diminue, certes légèrement, par rapport à la maison antéricure. Comparée à l'enrichissement de l'habitation. cette stagnation est particulièrement significative. A la fïn du II s.. l'eau devient une denrée onéreuse, ou bien passée de mode, dont l'utilisation, dans les bassins, est plus modérée que celle des placages de marbre.

$$
\therefore
$$

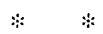

$1.38 \mathrm{Il}$ est peu probable que le portique ait été entièrement détruit. Dans la mesure où il reste l'aile oecidentale à desservir, la colomnade ouest conserve une utilité pratique ce qui n'est plus le cas des branches est et nord. devenues alors essentiellement décoratives. 
Le trottoir et le dallage de la rue du Commerce. d'une part. et les cing mosaïques de la maison. d'autre part. donnent la mesure de l'opulence de Vienne el de l'un de ses habitants, au début du III's. Cette image classique des bienfaits de la paix romaine n'est qu'un point d'orgue de l'histoire d'un site dont la variété des mutations constitue, de nos jours, la véritable richesse.

Notre perception du quartier s'affine au gré des nouvelles fouilles ou du réexamen des vestiges anciennement dégagés. En particulier, les traces éparses des premières occupations commencent à s'organiser en une trame cohérente d'où ressortent quelques fils conducteurs qui devraient guider les futures recherches. Il en va de la sorte pour la cadastration et les modes de construction. Ces derniers nous permettent de dégager certaines tendances et de formuler quelques hypothèses de développement. Ainsi, l'utilisation de fondations en galets, dans le troisième quart du $\mathrm{I}^{\mathrm{cr}} \mathrm{s}$. avant J.-C., semble encore relever d'une pratique indigène tandis que l'emploi fréquent de briques, dès le début du $\mathrm{I}^{\mathrm{er}} \mathrm{s}$. après J.-C., suggère une romanité intégrée ${ }^{139}$. Entre ces deux pôles, il convient de préciser la place occupée par les constructions qui utilisent un mortier blanc dans leurs maçonneries. Si beaucoup reste encore à faire, d'ores et déjà, il s'avère essentiel d'envisager, pour l'avenir, des fouilles d'envergure concernant les niveaux profonds. Une telle exploration nous apporterait de précieux renseignements sur l'organisation du site au $\mathrm{I}^{\mathrm{er}} \mathrm{s}$. avant J.-C. et contribuerait à établir le contexte géographique dans lequel s'effectue le passage de l'agglomération allobroge à la ville romaine ${ }^{140}$.

Si l'étude de la plupart des bâtiments dégagés reste à approfondir, la poursuite de l'analyse générale de la voirie devrait nous amener à une perception plus détaillée de l'évolution du quartier. Pour ce faire. l'affinement

1.39 A Saint-Romain-en-Gal, il existerait, au plus, un retard d'environ 80 ans par rapport à la date d'apparition de la brique dans les constructions de Campanic. Or. même en Italic, les exemples d'opus mixtum semblent rares au $\mathrm{I}^{\mathrm{er}} \mathrm{s}$. avant J.-C. (Adam. 1989, p. 152-154). Dans ces conditions, nous sommes en droit de penser que ce n'est qu'au début du $\mathrm{I}^{\mathrm{er}} \mathrm{s}$. après J.-C. que nous assistons à la généralisation de cette technique dont Lyon et Vienne ont profité. sans doute rapidement (Desbat, 1992). En effet, compte-tenu de l'importance de ces deux villes et de l'attention dont elles ont fait l'objet de la part de Rome, il semble peu probable que des nouveautés. techniques ou autres, mettent plus de quelques années pour être diffusées dès lors que la péninsule les a adoptées. Ainsi. les dates voisines de construction des maisons au Bassin Excentré et de la Fortune Annonaire montrent bien la contemporanéité d'apparition d'une forme architecturale à Vienne êt à Ostic.

140) Les données récentes, issues du chantier de fouilles des $n^{\text {os }}$ 25-31 de la rue de Bourgogne, ont renouvelé nos connaissances de la période "gauloise» de Vienne en révélant l'emplacement d'habitats et en précisant le contexte topographique du centre ville (Kroichvili. 1993). des chronologies relatives de chaque rue et de chaque bâtiment constitue une étape primordiale de la recherche sur le site, non seulement dans une perspective urbanistique mais aussi du point de vue des études céramologiques.

En effet. les datations basées sur les monnaies et la céramique sigillée montrent leurs limites lorsque les phases de construction se succèdent dans des intervalles de temps restreints. Seul l'établissement de faciès incluant tous les types de céramique et s'appuyant sur des regroupements fiables de couches peut prétendre pallier ce problème. Encore faut-il arriver à trouver une cohésion entre les données de fouilles et l'analyse céramologique. Ainsi, même si un parallèle a été établi entre les évolutions des parcelles des maisons aux Cinq Mosaiques et des Dieux Océans, sa cohérence reste à affiner, voire à confirmer pour certaines transitions. L'introduction de phases supplémentaires dans l'histoire de la parcelle de la maison des Dieux Océans nécessite de reprendre l'analyse chronologique de celle-ci. et rend indispensable une redéfinition des horizons à l'échelle de l'îlot. Pour cela, il conviendra également de préciser les événements qui ont affecté la parcelle de Petits Entrepôts. Nous disposerons alors d'un ensemble spatialement homogène qui permettra l'établissement de contextes céramologiques et archéologiques particulièrement fiables.

Jean-Luc PRISSET et Laurence BRISSAUD

\section{Nota Bene -}

M.-N. Baudrand* (* Equipe archéologique départementale) (fig. 25) : L. Brissaud* (fig. 1, 4.6 à 8.12 à 15, 16b. 20)-22, $26-32,36$ à $38,40-44,50,59,60,64,65 b, 68,69,72,75$. 79b. 80, 107): A. Canal (S.R.A.) (fig. 40, relevé): O. Leblanc* (fig. 81-105); V. Piccolo* (fig. 2) ; P. Platticr* (fig. 3, 11, 54) : J.-L. Prisset* (fig. 5. 9a. 10, 17. 18. 24. $35.39,45-47,51-53,56-59,61-63,65 \mathrm{a}, 67.68,72,74.79 \mathrm{c}-$ d. $80,106,108,109$ ): H. Savay-Guerraz* (fig. 9b, 33, 70. $71 \mathrm{~b})$ : Service régional de l'Archéologic (S.R.A.) (fig. 34. 71 a) : P. Veysseyre* (fig. 16a, 19, 23, 48, 49, 55, 66, 73, 76 à 79a, 110, ainsi que les prises de vue des monnaies. le tirage des bromures. C. Bassier el J. Prodhomme pour les dessins originaux des mosaïques. Toute notre gratitude à M. Salichon pour son remarquable travail de réduction sur les figures 43 et 72.

Nous tenons à exprimer nos remerciements à l'ensemble des stagiaires et terrassiers qui ont bravé les rayons ardents du soleil d'été et la moite fraîcheur des sondages pour qu'une petite maison sympathique retrouve son animation d'autrefois : $\mathrm{Z}$. Aggab, B. Ba. I. Bérault, L. Bontoux, A. P. Cardoso, M. Chamakoff-Depardon, N. Christodoulides, L. Combet. J. Cosson. D. Dufour, M. Dupessey, M. Ferrier. J. Germain. C. Gourdon, K. Harizia, Cl. Hiblot. H. Jannin. M.-H. Jeanjean. B. Juge, C. Jung, E. Lagrevol, H. Lalioui, L. Layachi ( + ). S. Lebrun, Fr. Lemarié, P. Maitre, Ph. Mancini, Cl. Marcellin. M.-R. Mas, B. Melhenas, J.-C. Pennec, B. Porcheron, K. Righi, G. Roy, M.-H. Sapin-Lévy, S. Saunier, R.-M. Solé. R. Spica, Y. Zoubaïda. 